Submitted to the Annals of Statistics

arXiv: arXiv: 1212.0478

\title{
SUPPLEMENTARY MATERIAL FOR: STRUCTURE ESTIMATION FOR DISCRETE GRAPHICAL MODELS: GENERALIZED COVARIANCE MATRICES AND THEIR INVERSES
}

\author{
By Po-Ling Loh, Martin J. Wainwright \\ University of California, Berkeley
}

\section{APPENDIX A: PROOFS OF SUPPORTING LEMMAS FOR THEOREM 1}

In this section, we supply the proofs of Lemmas 1 and 2, which are used in the proof of Theorem 1.

A.1. Proof of Lemma 1. By Proposition B.2 of Wainwright and Jordan [8] (cf. Theorems 23.5 and 26.3 of Rockafellar [7]), we know that the dual function $\Phi^{*}$ is differentiable on the interior of the marginal polytope $\mathcal{M}$ defined in equation (3.2), in particular with

$$
\nabla \Phi^{*}(\mu)=(\nabla \Phi)^{-1}(\mu) \text { for all } \mu \in \operatorname{int}(\mathcal{M}) .
$$

Also, by Theorem 3.4 of Wainwright and Jordan [8], for any $\mu \in \operatorname{int}(\mathcal{M})$, the negative dual function takes the form $\Phi^{*}(\mu)=-H\left(q_{\theta}(\mu)\right)$, where $\theta(\mu)=$ $(\nabla \Phi)^{-1}(\mu)$.

By relation (A.1), we have

$$
(\nabla \Phi)\left(\nabla \Phi^{*}(\mu)\right)=\mu \quad \text { for all } \mu \in \mathcal{M} .
$$

Since this equation holds on an open set, we may take derivatives; employing the chain rule yields

$$
\left(\nabla^{2} \Phi\right)\left(\nabla \Phi^{*}(\mu)\right) \cdot\left(\nabla^{2} \Phi^{*}(\mu)\right)=I_{D \times D} .
$$

Rearranging yields the relation $\nabla^{2} \Phi^{*}(\mu)=\left.\left(\nabla^{2} \Phi(\theta)\right)^{-1}\right|_{\theta=\theta(\mu)}$, as claimed.

A.2. Proof of Lemma 2. We induct on the subset size. For sets of size 1, the claim is obvious. Now suppose the claim holds for all subsets up to some size $k>1$, and consider a subset of size $k+1$, which we write as $C=\{1, \ldots, k+1\}$, without loss of generality. For any configuration 
$J \in \mathcal{X}_{0}^{|C|}$, the marginal probability $q_{C}\left(x_{C}=J\right)$ is equal to $\mu_{C ; J}$, by construction. Consequently, we need only specify how to determine the probabilities $q_{C}\left(x_{C}=J\right)$ for configurations $J \in \mathcal{X}^{|C|} \backslash \mathcal{X}_{0}^{|C|}$. By the definition of $\mathcal{X}_{0}^{|C|}$, each $j \in J$ has $j_{s}=0$ for at least one $s \in\{1, \ldots, k+1\}$.

We show how to express the remaining marginal probabilities sequentially, inducting on the number of positions $s$ for which $j_{s}=0$. Starting with the base case in which there is a single zero, suppose without loss of generality that $j_{k+1}=0$. For each $\ell \in\{1,2, \ldots, m-1\}$, let $J^{\ell}$ be the configuration such that $J_{i}^{\ell}=J_{i}$ for all $i \neq k+1$ and $J_{k+1}^{\ell}=\ell$. Defining $D:=C \backslash\{k+1\}$, we then have

$$
q_{C}\left(x_{C}=J\right)=q_{D}\left(x_{D}=J^{\prime}\right)-\sum_{\ell=1}^{m-1} q_{C}\left(x_{C}=J^{\ell}\right),
$$

where $J^{\prime} \in \mathcal{X}^{k}$ is the configuration defined by $J_{i}^{\prime}=J_{i}$ for all $i=1,2, \ldots, k$. Since $|D|=k$, our induction hypothesis implies that $q_{D}\left(x_{D}=J^{\prime}\right)$ is a linear function of the specified mean parameters. Moreover, our starting assumption implies that $J^{\ell} \in \mathcal{X}_{0}^{|C|}$ for all $\ell=\{1,2, \ldots, m-1\}$, so we have $q_{C}\left(x_{C}=J^{\ell}\right)=\mu_{C ; J^{\ell}}$. This establishes the base case.

Now suppose the sub-claim holds for all configurations with at most $t$ nonzeros, for some $t>1$. Consider a configuration $J$ with $t+1$ zero entries. Again without loss of generality, we may assume $j_{k+1}=0$, so equation (A.2) may be derived as before. This time, the configurations $J^{\ell}$ are not in $\mathcal{X}_{0}^{|C|}$ (since they still have $t \geq 1$ zero entries); however, our induction hypothesis implies that the corresponding probabilities may be written as functions of the given mean parameters. This completes the inductive proof of the inner claim, thereby completing the outer induction, as well.

\section{APPENDIX B: PROOFS OF POPULATION-LEVEL COROLLARIES}

In this Appendix, we prove Corollaries 1 and 3. (As previously noted, Corollary 2 is an immediate consequence of Corollary 1.)

B.1. Proof of Corollary 1. Recall that $\widetilde{\mathcal{C}}$ denotes the set of all cliques in the triangulation $\widetilde{G}$. The covariance matrix in Theorem 1 is indexed by $\widetilde{\mathcal{C}}$, and our goal is to define appropriate blocks of the matrix and then apply the matrix inversion lemma [3]. Consider the collection pow $(\mathcal{S})$. We define the collection of singleton subsets $V=\{\{1\},\{2\}, \ldots,\{p\}\}$, and introduce the disjoint partition

$$
\widetilde{\mathcal{C}}=\underbrace{(\operatorname{pow}(\mathcal{S}) \cup V)}_{\mathcal{U}} \cup \underbrace{(\widetilde{\mathcal{C} \backslash\{\operatorname{pow}(\mathcal{S}) \cup V\})} .}_{\mathcal{W}}
$$


The following property of the collection $\mathcal{W}$ is important:

Lemma 3. For each maximal clique $C \in \overline{\mathcal{C}}$, define the set collection $\mathcal{F}(C)=\operatorname{pow}(C) \backslash \mathcal{U}$. For any $A \in \mathcal{W}$, we have $A \in \mathcal{F}(C)$ for exactly one $C$.

Proof. We first establish existence. Since $\mathcal{W} \subseteq \widetilde{\mathcal{C}}$, any set $A \in \mathcal{W}$ is contained in some maximal clique $C_{A}$. Since $A \notin \mathcal{U}$, we clearly have $A \in$ $\mathcal{F}\left(C_{A}\right)$.

To establish uniqueness, consider a set $A$ belonging to the intersection $C_{1} \cap C_{2}$ of two maximal cliques. If these cliques are adjacent in the junction tree, then $A$ belongs to the separator set $C_{1} \cap C_{2}$, so $A$ cannot belong to $\mathcal{W}$, by definition. Even when $C_{1}$ and $C_{2}$ are not adjacent, the running intersection property of the junction tree implies that $C_{1} \cap C_{2}$ must belong to every separator set on the unique path between $C_{1}$ and $C_{2}$ in the junction tree, implying that $A \notin \mathcal{W}$, as before. This is a contradiction, implying that the maximal clique $C_{A}$ is unique.

Define $\Gamma=(\operatorname{cov}(\Psi(X ; \widetilde{\mathcal{C}})))^{-1}$. By the block-matrix inversion formula [3], we may write

$$
\Theta:=(\operatorname{cov}(\Psi(X ; \mathcal{U})))^{-1}=\Gamma(\mathcal{U}, \mathcal{U})-\Gamma(\mathcal{U}, \mathcal{W})(\Gamma(\mathcal{W}, \mathcal{W}))^{-1} \Gamma(\mathcal{W}, \mathcal{U})
$$

We need to show that $\Theta(A, B)=0$ for any members $A, B \in \mathcal{U}$ that do not belong to the same maximal clique. By Theorem $1(\mathrm{a})$, we have $\Gamma(A, B)=0$ whenever $A$ and $B$ do not belong to the same maximal clique, so it remains to show that $\Gamma(A, \mathcal{W})(\Gamma(\mathcal{W}, \mathcal{W}))^{-1} \Gamma(\mathcal{W}, B)=0$

We begin by observing that the matrix $\Gamma(\mathcal{W}, \mathcal{W})$ is block-diagonal with respect to the partition $\{\mathcal{F}(C): C \in \mathcal{C}\}$ previously defined in Lemma 3. (Indeed, consider two sets $D, E \in \mathcal{W}$ with $D \in \mathcal{F}(C)$ and $E \in \mathcal{F}\left(C^{\prime}\right)$ for distinct maximal cliques $C \neq C^{\prime}$. Two such sets cannot belong to the same maximal clique, so Theorem $1(\mathrm{a})$ implies that $\Gamma(D, E)=0$.) Since block-diagonal structure is preserved by matrix inversion, the inverse $\Upsilon=(\Gamma(\mathcal{W}, \mathcal{W}))^{-1}$ shares this property, so for any two members $A, B \in \mathcal{U}$, we may write

$$
\Gamma(A, \mathcal{W})(\Gamma(\mathcal{W}, \mathcal{W}))^{-1} \Gamma(\mathcal{W}, B)
$$

$$
=\sum_{\mathcal{F}(C), C \in \overline{\mathcal{C}}} \Gamma(A, \mathcal{F}(C)) \Upsilon(\mathcal{F}(C), \mathcal{F}(C)) \Gamma(\mathcal{F}(C), B) .
$$

We claim that each of these terms vanishes. For a given maximal clique $C^{\prime}$, suppose $A$ is not contained within $C^{\prime}$; we first claim that $\Gamma\left(A, \mathcal{F}\left(C^{\prime}\right)\right)=0$, 
or equivalently, for any set $D \in \mathcal{F}\left(C^{\prime}\right)$, we have $\Gamma(A, D)=0$. From Theorem 1(a), it suffices to show that $A$ and $D$ cannot be contained within the same maximal clique. From Lemma 3 , we know that $A$ belongs to a unique maximal clique $C$. Any set $D \in \mathcal{F}\left(C^{\prime}\right)$ is contained within $C^{\prime}$; if it were also contained within $C$, then $D$ would be contained in $C \cap C^{\prime}$. But as argued in the proof of Lemma 3, this implies that $D$ is contained within some separator set, whence it cannot belong to $\mathcal{F}\left(C^{\prime}\right)$. We thus conclude that $\Gamma(A, D)=0$, as claimed.

Taking any two subsets $A$ and $B$ that are not contained in the same maximal clique, we see that for any clique $C$, we must either have $\Gamma(A, \mathcal{F}(C))=0$ or $\Gamma(\mathcal{F}(C), B)=0$. Hence, each term in the sum (B.2) indeed vanishes, completing the proof.

B.2. Proof of Corollary 3. This corollary follows by a similar argument as in the proof of Corollary 1 . As before, let $\mathcal{C}$ denote the set of all cliques in the triangulation $\widetilde{G}$, and let $V=\{\{1\},\{2\}, \ldots,\{p\}\}$. Define $\mathcal{U}=\operatorname{pow}(\mathcal{S}(s ; d)) \cup V$ and $\mathcal{W}=\widetilde{\mathcal{C}} \backslash \mathcal{U}$.

Let $C_{s}:=s \cup N(s)$, and consider a disjoint partition of $\mathcal{W}$ defined by $\mathcal{F}_{1}:=\operatorname{pow}\left(C_{s}\right) \backslash \mathcal{U}$ and $\mathcal{F}_{2}:=\mathcal{W} \backslash \mathcal{F}_{1}$. Note that $C_{s}$ is the unique maximal clique in $\widetilde{\mathcal{C}}$ containing $s$. By construction, every clique in $\mathcal{F}_{2}$ does not contain $s$ and has more than $d$ elements, whereas every clique in $\mathcal{F}_{1}$ is contained in $C_{s}$, with $\left|C_{s}\right| \leq d+1$. It follows that no two cliques $A \in \mathcal{F}_{1}$ and $B \in \mathcal{F}_{2}$ can be contained in the same maximal clique. Denoting $\Gamma:=(\operatorname{cov}(\Psi(X ; \widetilde{\mathcal{C}})))^{-1}$, we conclude via Theorem $1(\mathrm{a})$ that $\Gamma(\mathcal{W}, \mathcal{W})$ is block-diagonal.

We now use the block matrix-equation formula (B.1). As before, Theorem 1 (a) implies that $\Gamma(\mathcal{U}, \mathcal{U})$ is graph-structured according to $\widetilde{G}$. In particular, for any $B \in \mathcal{U}$ with $B \subsetneq C_{s}$, we have $\Gamma(\{s\}, B)=0$. (The elements of $\mathcal{U}$ that are subsets of $C_{s}$ are exactly $\{s\}$ and the nonempty subsets of $N(s)$.) Hence, it remains to show that $\Gamma(\{s\}, \mathcal{W})(\Gamma(\mathcal{W}, \mathcal{W}))^{-1} \Gamma(\mathcal{W}, B)=0$.

Analogous to equation (B.2), we may write

$$
\Gamma(\{s\}, \mathcal{W})(\Gamma(\mathcal{W}, \mathcal{W}))^{-1} \Gamma(\mathcal{W}, B)=\sum_{i=1}^{2} \Gamma\left(\{s\}, \mathcal{F}_{i}\right) \Upsilon\left(\mathcal{F}_{i}, \mathcal{F}_{i}\right) \Gamma\left(\mathcal{F}_{i}, B\right)
$$

where $\Upsilon:=(\Gamma(\mathcal{W}, \mathcal{W}))^{-1}$. Applying Theorem 1(a) once more, we see that $\Gamma\left(\mathcal{F}_{1}, B\right)=0$, since $B \subsetneq C_{s}$ and $\Gamma\left(\{s\}, \mathcal{F}_{2}\right)=0$. Therefore, the matrix $\Theta=(\operatorname{cov}(\Psi(X ; \mathcal{U})))^{-1}$ appearing in equation (B.1) is indeed $s$-block graphstructured. 


\section{APPENDIX C: PROOF OF PROPOSITION 1}

In this section, we provide a proof of our main nodewise recovery result, Proposition 1. For proofs of supporting technical lemmas and all corollaries appearing in the text, see Appendix D.

C.1. Main argument. We derive Proposition 1 as a consequence of a more general theorem. Suppose we have i.i.d. observations $\left\{\left(x_{i}, y_{i}\right)\right\}_{i=1}^{n}$, with $x_{i} \in \mathbb{R}^{p}$ and $y_{i} \in \mathbb{R}$, and we wish to estimate the best linear predictor $\widetilde{\beta}=\Sigma_{x}^{-1} \operatorname{Cov}\left(x_{i}, y_{i}\right)$, when $\widetilde{\beta}$ is $k$-sparse. Loh and Wainwright [5] formulated a modified version of the Lasso based on possibly corrupted observations; however, they assume the linear regression model

$$
y_{i}=x_{i}^{T} \widetilde{\beta}+\epsilon_{i},
$$

where $\epsilon_{i}$ is sub-Gaussian noise and $\epsilon_{i} \Perp x_{i}$. Although the model (C.1) holds in the case where $y_{i}$ is a sample from a single node and $x_{i}$ is a sample from all other nodes in a Gaussian graphical model, the model (C.1) does not hold in a general discrete graphical model. Nonetheless, we show that essentially the same Lasso estimator provides an estimator for $\widetilde{\beta}$ that is consistent for support recovery. Suppose the pair $(\widehat{\Gamma}, \widehat{\gamma})$ in the Lasso program (4.6) satisfies the following deviation bounds:

$$
\begin{gathered}
\|\widehat{\Gamma} \widetilde{\beta}-\widehat{\gamma}\|_{\infty} \leq \varphi_{1} \sqrt{\frac{\log p}{n}}, \\
\left\|\left(\widehat{\Gamma}-\Sigma_{x}\right) v\right\|_{\infty} \leq \varphi_{2}\|v\|_{\infty} \sqrt{\frac{k \log p}{n}} \quad \forall v \in \mathbb{B}_{1}(8 k) \cap \mathbb{B}_{\infty}(1),
\end{gathered}
$$

for some $\varphi_{1}, \varphi_{2}$. Also suppose $\widehat{\Gamma}$ satisfies the lower-restricted eigenvalue (RE) condition:

$$
v^{T} \widehat{\Gamma} v \geq \alpha\|v\|_{2}^{2} \quad \forall v \text { s.t. }\|v\|_{1} \leq \sqrt{k}\|v\|_{2} .
$$

Then we have the following technical result:

THEOREM 2. Suppose the pair $(\widehat{\Gamma}, \widehat{\gamma})$ satisfies the deviation conditions (C.2a) and (C.2b), as well as the lower-RE condition (C.3). Also suppose $n \succsim \max \left\{\frac{\varphi_{1}^{2}}{\alpha^{2}\left(b_{0}-\|\widetilde{\beta}\|_{2}\right)^{2}}, \varphi_{2}^{2}\left\|\Sigma_{x}^{-1}\right\|_{\infty}^{2}\right\} k \log p$ and $\lambda_{n} \succsim \varphi_{1} \sqrt{\frac{\log p}{n}}$. Then any optimum $\widehat{\beta}$ of the Lasso program (4.6) satisfies

$$
\|\widehat{\beta}-\widetilde{\beta}\|_{\infty} \leq 4 \lambda_{n}\left\|\Sigma_{x}^{-1}\right\|_{\infty} .
$$


The proof of Theorem 2 is provided in Appendix C.2. In order to prove Proposition 1, we first establish that the deviation conditions (C.2a) and (C.2b) of Theorem 2 hold w.h.p. with $\left(\varphi_{1}, \varphi_{2}\right)=\left(\varphi\|\widetilde{\beta}\|_{2}, \varphi\right)$, and the lowerRE condition holds with $\alpha=\frac{1}{2} \lambda_{\min }\left(\Sigma_{x}\right)$.

Note that

$$
\|\widehat{\Gamma} \widetilde{\beta}-\widehat{\gamma}\|_{\infty} \leq\left\|\left(\widehat{\Gamma}-\Sigma_{x}\right) \widetilde{\beta}\right\|_{\infty}+\left\|\operatorname{Cov}\left(x_{i}, y_{i}\right)-\widehat{\gamma}\right\|_{\infty} .
$$

Furthermore,

$$
\left\|\left(\widehat{\Gamma}-\Sigma_{x}\right) \widetilde{\beta}\right\|_{\infty} \leq\left\|\left(\frac{X^{T} X}{n}-\mathbb{E}\left(x_{i} x_{i}^{T}\right)\right) \widetilde{\beta}\right\|_{\infty}+\left\|\left(\bar{x} \bar{x}^{T}-\Sigma_{x}\right) \widetilde{\beta}\right\|_{\infty}
$$

and

$$
\left\|\operatorname{Cov}\left(x_{i}, y_{i}\right)-\widehat{\gamma}\right\|_{\infty} \leq\left\|\frac{X^{T} y}{n}-\mathbb{E}\left(y_{i} x_{i}\right)\right\|_{\infty}+\left\|\bar{y} \bar{x}-\mathbb{E}\left(y_{i}\right) \mathbb{E}\left(x_{i}\right)\right\|_{\infty} .
$$

As in the analysis of inequality (E.1) below, we may disregard the two second terms involving empirical means, since they concentrate at a fast rate. Since $x_{i}^{T} \widetilde{\beta}$ is sub-Gaussian with parameter $\varphi^{2}\|\widetilde{\beta}\|_{2}^{2}$ by assumption, and $e_{j}^{T} x_{i}$ and $y_{i}$ are clearly sub-Gaussian with parameter 1, the deviation condition (C.2a) follows with $\varphi_{1}=\varphi\|\widetilde{\beta}\|_{2}$ by standard concentration bounds on an i.i.d. average of products of sub-Gaussians (cf. Lemma 14 of Loh and Wainwright [5]).

For the second deviation bound, we will verify the bound over a more tractable set via the following lemma:

Lemma 4. For any constant $c_{0}>0$, we have

$$
\mathbb{B}_{1}\left(c_{0} k\right) \cap \mathbb{B}_{\infty}(1) \subseteq\left(1+c_{0}\right) \operatorname{cl}\left\{\operatorname{conv}\left\{\mathbb{B}_{0}(k) \cap \mathbb{B}_{\infty}(1)\right\}\right\} .
$$

Hence, it is sufficient to establish the deviation inequality (C.2b) over the set $\mathbb{B}_{0}(k) \cap \mathbb{B}_{\infty}(1)$. We proceed via a discretization argument. Suppose $\left\{v_{1}, \ldots, v_{M}\right\}$ is a $\frac{1}{2}$-covering of the unit $\ell_{\infty}$-ball in $\mathbb{R}^{k}$ in its own metric. By standard results on metric entropy, we know that such a covering exists with $M \leq c^{k}$. Writing $\psi(v)=\left\|\left(\widehat{\Gamma}-\Sigma_{x}\right) v\right\|_{\infty}$, we know that there exists $v_{j}$ such that $\left\|v-v_{j}\right\|_{\infty} \leq \frac{1}{2}$. Let $\Delta v=v-v_{j}$. Then

$\psi(v)=\left\|\left(\widehat{\Gamma}-\Sigma_{x}\right)\left(v_{j}+\Delta v\right)\right\|_{\infty} \leq \psi\left(v_{j}\right)+\psi(\Delta v) \leq \sup _{1 \leq j \leq M} \psi\left(v_{j}\right)+\frac{1}{2} \sup _{\|v\|_{\infty} \leq 1} \psi(v)$,

simply by rescaling. Taking the sup over $\left\{\|v\|_{\infty} \leq 1\right\}$ on the LHS and rearranging then yields

$$
\sup _{\|v\|_{\infty} \leq 1} \leq 2 \sup _{1 \leq j \leq M} \psi\left(v_{j}\right)
$$


Hence, it suffices to establish the bound for a given $v \in \mathbb{B}_{1}\left(c_{0} k\right) \cap \mathbb{B}_{\infty}(1)$, then take a union bound over the $M \leq c^{k}$ elements in the discretization and the $\left(\begin{array}{l}p \\ k\end{array}\right) \leq p^{k}$ choices of the support set.

For a given $k$-sparse $v$, note that $x_{i}^{T} v$ has sub-Gaussian parameter $\varphi^{2}\|v\|_{2}^{2}$ by assumption, and

$$
\|v\|_{2}^{2} \leq\|v\|_{1}\|v\|_{\infty} \leq \sqrt{k}\|v\|_{2}\|v\|_{\infty}
$$

so $x_{i}^{T} v$ is sub-Gaussian with parameter $\varphi^{2} k\|v\|_{\infty}^{2}$. Since $e_{\ell}^{T} x_{i}$ is sub-Gaussian with parameter 1 , it follows from the same recentering techniques as in inequality (E.1) that

$$
\left\|\left(\widehat{\Gamma}-\Sigma_{x}\right) v\right\|_{\infty}=\max _{\ell}\left|e_{\ell}^{T}\left(\widehat{\Gamma}-\Sigma_{x}\right) v\right| \leq t,
$$

with probability at least $1-c_{1} \exp \left(\frac{-c_{2} n t^{2}}{\varphi^{2} k\|v\|_{\infty}^{2}}\right)$. Taking a union bound over the discretization and setting $t=c \varphi \sqrt{k}\|v\|_{\infty} \sqrt{\frac{k \log p}{n}}$ then implies the deviation bound (C.2b) with $\varphi_{2}=\varphi$, under the scaling $n \succsim \varphi^{2} k^{2} \log p$.

The lower-RE condition (C.3) may be verified analogously to the results in Loh and Wainwright [5]. The only difference is to use the fact that $x_{i}^{T} v$ is sub-Gaussian with parameter $\varphi^{2}\|v\|_{2}^{2}$ in all the deviation bounds. Then the lower-RE condition holds with probability at least $1-c_{1} \exp \left(-c_{2} k \log p\right)$, under the scaling $n \succsim \varphi^{2} k \log p$.

We may take $\lambda_{n} \asymp \varphi\|\widetilde{\beta}\|_{2} \sqrt{\frac{\log p}{n}}$ in Theorem 2 to conclude that w.h.p.,

$$
\|\widehat{\beta}-\widetilde{\beta}\|_{\infty} \precsim \varphi \mid \widetilde{\beta} \|_{2} \sqrt{\frac{\log p}{n}} .
$$

Finally, note that the vector $\widetilde{\beta}$ is a scalar multiple of column $s$ of the inverse covariance matrix $\Gamma$, as a straightforward consequence of block matrix inversion. Hence, combining Corollary 1 and Theorem 2 implies that thresholding succeeds w.h.p. for neighborhood recovery in a tree graph.

C.2. Proof of Theorem 2. We begin by establishing $\ell_{1^{-}}$and $\ell_{2^{-}}$error bounds, which will be used in the sequel:

Lemma 5. Suppose the deviation condition (C.2a) holds and $\widehat{\Gamma}$ satisfies the lower-RE condition (C.3). Also suppose $\lambda_{n} \succsim \varphi_{1} \sqrt{\frac{\log p}{n}}$. Then any global 
optimum $\widehat{\beta}$ of the Lasso program (4.6) satisfies the bounds

$$
\begin{aligned}
& \|\widehat{\beta}-\widetilde{\beta}\|_{2} \leq \frac{c_{0} \sqrt{k}}{\alpha_{\ell}} \max \left\{\varphi_{1} \sqrt{\frac{\log p}{n}}, \lambda_{n}\right\}, \\
& \|\widehat{\beta}-\widetilde{\beta}\|_{1} \leq \frac{8 c_{0} k}{\alpha_{\ell}} \max \left\{\varphi_{1} \sqrt{\frac{\log p}{n}}, \lambda_{n}\right\} .
\end{aligned}
$$

We now argue that for suitable scaling $n \succsim k \log p$, any optimum $\widehat{\beta}$ lies in the interior of $\mathbb{B}_{1}\left(b_{0} \sqrt{k}\right)$ :

Lemma 6. Suppose $\widehat{\beta}$ is an optimum of the Lasso program (4.6). Then under the scaling $n \succsim\left(\frac{\varphi_{1}}{\alpha\left(b_{0}-\|\widetilde{\beta}\|_{2}\right)}\right)^{2} k \log p$, we have

$$
\widehat{\beta} \notin \partial \mathbb{B}_{1}\left(b_{0} \sqrt{k}\right) \text {. }
$$

By Lemma 6 , we are guaranteed that $\widehat{\beta}$ is an interior point of the feasible set. Consequently, by Proposition 2.3.2 of Clarke [2], we are guaranteed that 0 is a generalized gradient of the objective function at $\widehat{\beta}$. By Proposition 2.3.3 of Clarke [2], there must exist a vector $\left.\hat{z} \in \partial\|\beta\|_{1}\right|_{\beta=\widehat{\beta}}$ such that

$$
\widehat{\Gamma} \widehat{\beta}-\widehat{\gamma}+\lambda_{n} \hat{z}=0
$$

Denoting the loss function $\mathcal{L}(\beta)=\frac{1}{2} \beta^{T} \widehat{\Gamma} \beta-\widehat{\gamma}^{T} \beta$, we have $\nabla \mathcal{L}(\beta)=\widehat{\Gamma} \beta-\widehat{\gamma}$, so

$$
\nabla \mathcal{L}(\widetilde{\beta})-\nabla \mathcal{L}(\widehat{\beta})=\nabla \mathcal{L}(\widetilde{\beta})+\lambda_{n} \hat{z}=\widehat{\Gamma} \widetilde{\beta}-\widehat{\gamma}+\lambda_{n} \hat{z}
$$

Then

$$
\|\nabla \mathcal{L}(\widetilde{\beta})-\nabla \mathcal{L}(\widehat{\beta})\|_{\infty} \leq\|\widehat{\Gamma} \widetilde{\beta}-\widehat{\gamma}\|_{\infty}+\lambda_{n}\|\hat{z}\|_{\infty} \leq\|\widehat{\Gamma} \widetilde{\beta}-\widehat{\gamma}\|_{\infty}+\lambda_{n}
$$

Using the deviation bound (C.2a) again, we have

$$
\|\widehat{\Gamma} \widetilde{\beta}-\widehat{\gamma}\|_{\infty} \leq \varphi_{1} \sqrt{\frac{\log p}{n}} .
$$

It follows from equation (C.7) that if $\lambda_{n} \geq \varphi_{1} \sqrt{\frac{\log p}{n}}$, then

$$
\|\nabla \mathcal{L}(\widetilde{\beta})-\nabla \mathcal{L}(\widehat{\beta})\|_{\infty} \leq 2 \lambda_{n} .
$$

Finally, we lower-bound

$$
\begin{aligned}
\|\nabla \mathcal{L}(\widetilde{\beta})-\nabla \mathcal{L}(\widehat{\beta})\|_{\infty} & =\|\widehat{\Gamma} \widehat{\nu}\|_{\infty} \\
& \geq\left\|\Sigma_{x} \widehat{\nu}\right\|_{\infty}-\left\|\left(\widehat{\Gamma}-\Sigma_{x}\right) \widehat{\nu}\right\|_{\infty} \\
& \geq\left\|\Sigma_{x}^{-1}\right\|_{\infty}^{-1}\|\widehat{\nu}\|_{\infty}-\left\|\left(\widehat{\Gamma}-\Sigma_{x}\right) \widehat{\nu}\right\|_{\infty}
\end{aligned}
$$


Now note that $\|\widehat{\nu}\|_{1} \leq 8 \sqrt{k}\|\widehat{\nu}\|_{2}$, as shown in Loh and Wainwright [5], so we have

$$
\|\widehat{\nu}\|_{2}^{2} \leq\|\widehat{\nu}\|_{\infty}\|\widehat{\nu}\|_{1} \leq 8 \sqrt{k}\|\widehat{\nu}\|_{\infty}\|\widehat{\nu}\|_{2}
$$

so $\|\widehat{\nu}\|_{2} \leq 8 \sqrt{k}\|\widehat{\nu}\|_{\infty}$. In particular, $\|\widehat{\nu}\|_{1} \leq 8 k\|\widehat{\nu}\|_{\infty}$. Applying inequality $(\mathrm{C} .2 \mathrm{~b})$ to $v=\frac{\widehat{\nu}}{\|\widehat{\nu}\|_{\infty}}$ then gives

$$
\left\|\left(\widehat{\Gamma}-\Sigma_{x}\right) \widehat{\nu}\right\|_{\infty} \leq c \varphi_{2}\|\widehat{\nu}\|_{\infty} \sqrt{\frac{k \log p}{n}} .
$$

Combining this with inequality (C.9), we have

$$
\|\widehat{\Gamma} \widehat{\nu}\|_{\infty} \geq\|\widehat{\nu}\|_{\infty}\left(\frac{1}{\left\|\Sigma_{x}^{-1}\right\|_{\infty}}-c \varphi_{2} \sqrt{\frac{k \log p}{n}}\right),
$$

so when $n \succsim \varphi_{2}^{2}\left\|\left|\Sigma_{x}^{-1}\right|\right\|_{\infty}^{2} k \log p$, we have

$$
\|\widehat{\Gamma} \widehat{\nu}\|_{\infty} \geq \frac{1}{2} \frac{\|\widehat{\nu}\|_{\infty}}{\left\|\Sigma_{x}^{-1}\right\|_{\infty}} .
$$

Finally, combining with inequality (C.8) yields the result of the theorem.

\section{APPENDIX D: PROOF OF SUPPORTING LEMMAS TO PROPOSITION 1}

D.1. Proof of Lemma 4. We denote the left-hand set by $A$ and the right-hand set by $B$. It suffices to show that $\varphi_{A}(z) \leq \varphi_{B}(z)$ for all $z$, where $\varphi$ is the support function.

For a given $z$, let $S$ be the set of indices of coordinates of $z$ with highest absolute value. We may write

$$
\begin{aligned}
\varphi_{A}(z) & =\sup _{\theta \in A}\langle\theta, z\rangle \\
& =\sup _{\theta \in A}\left\langle\theta_{S}, z_{S}\right\rangle+\left\langle\theta_{S^{c}}, z_{S^{c}}\right\rangle \\
& \leq\left\|z_{S}\right\|_{1}+c_{0} k\left\|z_{S^{c}}\right\|_{\infty},
\end{aligned}
$$

since

$$
\left\langle\theta_{S}, z_{S}\right\rangle \leq\left\|\theta_{S}\right\|_{\infty}\left\|z_{S}\right\|_{1} \leq\|\theta\|_{\infty}\left\|z_{S}\right\|_{1} \leq\left\|z_{S}\right\|_{1}
$$

and

$$
\left\langle\theta_{S^{c}}, z_{S^{c}}\right\rangle \leq\left\|\theta_{S^{c}}\right\|_{1}\left\|z_{S^{c}}\right\|_{\infty} \leq c_{0} k\left\|z_{S^{c}}\right\|_{\infty}
$$

for $\theta \in A$. Furthermore, $k\left\|z_{S^{c}}\right\|_{\infty} \leq\left\|z_{S}\right\|_{1}$. Hence, inequality (D.1) becomes

$$
\varphi_{A}(z) \leq\left(1+c_{0}\right)\left\|z_{S}\right\|_{1} .
$$


Finally, note that

$$
\varphi_{B}(z)=\left(1+c_{0}\right) \max _{|U| \leq k} \sup _{\left\|\theta_{U}\right\|_{\infty} \leq 1}\left\langle\theta_{U}, z_{U}\right\rangle=\left(1+c_{0}\right)\left\|z_{S}\right\|_{1},
$$

establishing the desired result.

D.2. Proof of Lemma 5. The proof is essentially the same as in the case of a standard linear model [5]. From the fact that $\widetilde{\beta}$ is feasible and $\widehat{\beta}$ is optimal, we obtain a basic inequality. Furthermore, defining $\widehat{\nu}=\widehat{\beta}-\widetilde{\beta}$, we may verify the cone condition $\|\widehat{\nu}\|_{1} \leq c \sqrt{k}\|\widehat{\nu}\|_{2}$. We will not repeat the arguments here.

D.3. Proof of Lemma 6. Note that

$$
\|\widehat{\beta}-\widetilde{\beta}\|_{1} \geq\|\widehat{\beta}\|_{1}-\|\widetilde{\beta}\|_{1} \geq\|\widehat{\beta}\|_{1}-\sqrt{k}\|\widetilde{\beta}\|_{2} .
$$

Hence, if $\widehat{\beta} \in \partial \mathbb{B}_{1}\left(b_{0} \sqrt{k}\right)$, we have

$$
\|\widehat{\beta}-\widetilde{\beta}\|_{1} \geq b_{0} \sqrt{k}-\|\widetilde{\beta}\|_{2} \sqrt{k}=\left(b_{0}-\|\widetilde{\beta}\|_{2}\right) \sqrt{k} .
$$

On the other hand, Theorem 1 in Loh and Wainwright [5] guarantees that under deviation condition (C.2a) and the lower-RE condition (C.3), we have the $\ell_{1}$-bound

$$
\|\widehat{\beta}-\widetilde{\beta}\|_{1} \leq \frac{c \varphi_{1} k}{\alpha} \sqrt{\frac{\log p}{n}} .
$$

Combining inequalities (D.2) and (D.3) gives

$$
\left(b_{0}-\|\widetilde{\beta}\|_{2}\right) \sqrt{k} \leq \frac{c \varphi_{1} k}{\alpha} \sqrt{\frac{\log p}{n}},
$$

contradicting the assumption that $n>\left(\frac{c \varphi_{1}}{\alpha\left(b_{0}-\|\tilde{\beta}\|_{2}\right)}\right)^{2} k \log p$.

\section{APPENDIX E: PROOFS OF SAMPLE-BASED COROLLARIES}

Here, we provide proofs for the remaining corollaries involved in samplebased approaches to graph selection. 
E.1. Proof of Corollary 4. As noted by Liu et al. [4], the proof of this corollary hinges only on the deviation condition condition (4.2) being satisfied w.h.p.; the rest of the proof follows from the analysis of Ravikumar et al. [6]. We verify inequality (4.2) with $\varphi\left(\Sigma^{*}\right)=c_{1}$ and $\psi(n, p)=c^{\prime} \log p$.

Note that

$$
\begin{aligned}
\|\widehat{\Sigma}-\Sigma\|_{\max } & =\left\|\left(\frac{1}{n} \sum_{i=1}^{n} x_{i} x_{i}^{T}-\bar{x} \bar{x}^{T}\right)-\Sigma\right\|_{\max } \\
& \leq\left\|\frac{1}{n} \sum_{i=1}^{n} x_{i} x_{i}^{T}-\mathbb{E}\left(x_{i} x_{i}^{T}\right)\right\|_{\max }+\left\|\bar{x} \bar{x}^{T}-\mathbb{E}\left(x_{i}\right) \mathbb{E}\left(x_{i}\right)^{T}\right\|_{\max },
\end{aligned}
$$

where we have used the triangle inequality and the fact that $\Sigma=\mathbb{E}\left(x_{i} x_{i}^{T}\right)$ $\mathbb{E}\left(x_{i}\right) \mathbb{E}\left(x_{i}\right)^{T}$ in the second line. Noting that $\|Y\|_{\max }=\max _{j, k}\left|e_{j}^{T} Y e_{k}\right|$ for a matrix $Y$, and the random variables $e_{j}^{T} x_{i}$ are i.i.d. Bernoulli (sub-Gaussian parameter 1) for each fixed $j$, we conclude by standard sub-Gaussian tail bounds (cf. Lemma 14 in Loh and Wainwright [5]) that the first term is bounded by $\sqrt{\frac{\log p}{n}}$, with probability at least $1-c \exp \left(-c^{\prime} \log p\right)$. For the second term, we may further bound

$$
\begin{array}{r}
\left\|\bar{x} \bar{x}^{T}-\mathbb{E}\left(x_{i}\right) \mathbb{E}\left(x_{i}\right)^{T}\right\|_{\max } \leq\left\|\left(\bar{x}-\mathbb{E}\left(x_{i}\right)\right)\left(\bar{x}-\mathbb{E}\left(x_{i}\right)\right)^{T}\right\|_{\max } \\
+2\left\|\mathbb{E}\left(x_{i}\right)\right\|_{\infty}\left\|\bar{x}-\mathbb{E}\left(x_{i}\right)\right\|_{\infty},
\end{array}
$$

by way of the triangle inequality. Note that $e_{j}^{T}\left(\bar{x}-\mathbb{E}\left(x_{i}\right)\right)$ is an average of i.i.d. sub-Gaussian variables with parameter 1 , hence has sub-Gaussian parameter $\frac{1}{n}$. Therefore, we have the even tighter bound $\frac{1}{n} \sqrt{\frac{\log p}{n}}$ for this term. Combining the bounds for the two terms in inequality (E.1) establishes the deviation condition (4.2).

By the machinery of Ravikumar et al. [6], we then have the elementwise bound

$$
\mathbb{P}\left[\left\|\widehat{\Theta}-\Theta^{*}\right\|_{\max } \geq \tau_{n}\right] \leq c \exp \left(-c^{\prime} \log p\right) .
$$

The statement about thresholding $\widehat{\Theta}$ to obtain a consistent estimate of $\Theta^{*}$ follows immediately.

E.2. Proof of Corollary 5. The analysis borrows techniques from the paper [1]. We first prove that under the scaling $n \succsim \kappa^{2} \log p$, we have $\left|r_{C}(s, t)-\widehat{r}_{C}(s, t)\right| \leq \frac{\kappa}{4}$ for all $(s, t) \in V \times V$, with probability at least $1-c_{1} \exp \left(-c_{2} \log p\right)$. First fix a pair $(s, t)$ and a corresponding pair of values $\left(x_{s}, x_{t}\right)$. By a simple application of Hoeffding's inequality, we have

$$
\mathbb{P}\left(\left|\mathbb{P}\left(X_{s}=x_{s}, X_{t}=x_{t}\right)-\widehat{\mathbb{P}}\left(X_{s}=x_{s}, X_{t}=x_{t}\right)\right| \geq \epsilon\right) \leq c \exp \left(-c^{\prime} n \epsilon^{2}\right),
$$


and similarly for the marginal deviations $\left|\mathbb{P}\left(X_{s}=x_{s}\right)-\widehat{\mathbb{P}}\left(X_{s}=x_{s}\right)\right|$ and $\left|\mathbb{P}\left(X_{t}=x_{t}\right)-\widehat{\mathbb{P}}\left(X_{t}=x_{t}\right)\right|$. Note that

$$
\begin{aligned}
\left|r_{C}(s, t)-\widehat{r}_{C}(s, t)\right| \leq \sum_{x_{s}, x_{t}}\left(\left|\mathbb{P}\left(X_{s}=x_{s}, X_{t}=x_{t}\right)-\widehat{\mathbb{P}}\left(X_{s}=x_{s}, X_{t}=x_{t}\right)\right|\right. \\
\left.\quad+\left|\mathbb{P}\left(X_{s}=x_{s}\right) \mathbb{P}\left(X_{t}=x_{t}\right)-\widehat{\mathbb{P}}\left(X_{s}=x_{s}\right) \widehat{\mathbb{P}}\left(X_{t}=x_{t}\right)\right|\right) .
\end{aligned}
$$

Furthermore,

$$
\begin{aligned}
\left|\mathbb{P}\left(X_{s}=x_{s}\right) \mathbb{P}\left(X_{t}=x_{t}\right)-\widehat{\mathbb{P}}\left(X_{s}=x_{s}\right) \widehat{\mathbb{P}}\left(X_{t}=x_{t}\right)\right| \\
\leq\left|\mathbb{P}\left(X_{s}=x_{s}\right)-\widehat{\mathbb{P}}\left(X_{s}=x_{s}\right)\right| \cdot \mathbb{P}\left(X_{t}=x_{t}\right) \\
\quad \quad+\left|\mathbb{P}\left(X_{t}=x_{t}\right)-\widehat{\mathbb{P}}\left(X_{t}-x_{t}\right)\right| \cdot \widehat{\mathbb{P}}\left(X_{s}=x_{s}\right) \\
\leq 2 \epsilon,
\end{aligned}
$$

so taking a union bound over all pairs $(s, t)$ and all values $\left(x_{s}, x_{t}\right)$, we have $\left|r_{C}(s, t)-\widehat{r}_{C}(s, t)\right| \leq 3 m^{2} \epsilon$ for all $(s, t) \in V \times V$, with probability at least $1-c m^{2} p^{2} \exp \left(-c^{\prime} n \epsilon^{2}\right)$. Finally, taking $\epsilon=\frac{\kappa}{12 m^{2}}$ and using the fact that $n \succsim \kappa^{2} \log p$ gives the desired bound, with probability at least $1-c_{1} \exp \left(-c_{2} \log p\right)$.

In particular, it follows that

$$
N(s) \subseteq \mathcal{C} \subseteq\left\{t \in V: r_{C}(s, t) \geq \frac{\kappa}{4}\right\}
$$

with probability at least $1-c_{1} \exp \left(-c_{2} \log p\right)$. Since the last subset has cardinality at most $d^{\frac{\log (4 / \kappa)}{\zeta}}$ by the correlation decay condition, we also have $|\mathcal{C}| \leq d^{\frac{\log (4 / \kappa)}{\zeta}}$, as claimed.

The remainder of the proof is identical to the proof of Proposition 1, and is a consequence of Theorem 2 .

\section{REFERENCES}

[1] G. Bresler, E. Mossel, and A. Sly. Reconstruction of Markov random fields from samples: Some observations and algorithms. In APPROX-RANDOM, pages 343-356, 2008.

[2] F. H. Clarke. Optimization and Nonsmooth Analysis. Wiley-Interscience, New York, 1983.

[3] R. A. Horn and C. R. Johnson. Matrix Analysis. Cambridge University Press, 1990.

[4] H. Liu, F. Han, M. Yuan, J.D. Lafferty, and L.A. Wasserman. High-dimensional semiparametric Gaussian copula graphical models. Annals of Statistics, 40(4):22932326, 2012. 
[5] P. Loh and M.J. Wainwright. High-dimensional regression with noisy and missing data: Provable guarantees with non-convexity. Annals of Statistics, 40(3):1637-1664, 2012 .

[6] P. Ravikumar, M. J. Wainwright, G. Raskutti, and B. Yu. High-dimensional covariance estimation by minimizing $\ell_{1}$-penalized log-determinant divergence. Electronic Journal of Statistics, 4:935-980, 2011.

[7] R. T. Rockafellar. Convex Analysis. Princeton University Press, Princeton, 1970.

[8] M. J. Wainwright and M. I. Jordan. Graphical models, exponential families, and variational inference. Found. Trends Mach. Learn., 1(1-2):1-305, January 2008. ISSN 1935-8237. 
Department of Statistics

University of California, Berkeley

Berkeley, California 94720

USA

E-MAIL: ploh@berkeley.edu wainwrig@stat.berkeley.edu 